\title{
Association of Autism Spectrum Disorder and Attention Deficit Hyperactivity Disorder Traits with Depression and Empathy Among Medical Students
}

\author{
Takafumi Watanabe (D) \\ Masaki Kondo iD \\ Mie Sakai ${ }^{2}$ \\ So Takabatake' \\ Toshiaki A Furukawa ${ }^{3}$ \\ Tatsuo Akechi' \\ 'Department of Psychiatry, Nagoya City \\ University Graduate School of Medical \\ Sciences, Nagoya City, Aichi, Japan; \\ ${ }^{2}$ Department of Anesthesiology and \\ Intensive Care Medicine, Nagoya City \\ University Graduate School of Medical \\ Sciences, Nagoya City, Aichi, Japan; \\ ${ }^{3}$ Department of Health Promotion and \\ Human Behavior, Kyoto University \\ Graduate School of Medicine/School of \\ Public Health, Kyoto City, Japan
}

\begin{abstract}
Purpose: This study aimed to investigate the associations of the traits of autism spectrum disorder (ASD) and attention deficit hyperactivity disorder (ADHD) with depression and empathy among medical students.
\end{abstract}

Patients and Methods: A cross-sectional survey was conducted with 202 fifth-year students at a Japanese medical school for 10 months during their clinical clerkship. The survey included sociodemographic questions and validated tools to measure depressive symptoms (Hospital Anxiety and Depression Scale [HADS]), medical students' empathy for patients (Jefferson Scale of Empathy-Student version [JSE]), ADHD traits (ADHD SelfReport Scale Screener [ASRS Screener]), and ASD traits (Autism-Spectrum Quotient Japanese version-21 [AQ-J-21]).

Results: A total of 151 students (response rate: 74.7\%) participated in the survey. Of these, $41(27.2 \%)$ reported a total score of $\geq 20$ on the HADS and were categorized as depressed. Depressed students reported significantly lower and higher rates of having a part-time job and a history of enrolment in other faculties, respectively, than non-depressed students. According to the cutoff criteria of the ASRS Screener and AQ-J-21, 31 (20.5\%) and 42 (27.8\%) students reported ADHD and ASD traits, respectively. Multivariate regression analysis, controlling for age and sex, reported that higher age, ASRS Screener scores, and AQ-J-21 scores were significant predictors of higher HADS total scores. Additionally, higher AQ-J-21 scores significantly predicted lower JSE scores.

Conclusion: The degree of ADHD and ASD traits was significantly associated with depression. Moreover, the degree of ASD traits was significantly associated with lower empathy for their patients. It is important to consider that about $20-30 \%$ of medical students have these neurodevelopmental traits and to develop intervention strategies for improving depression and empathy.

Keywords: medical education, neurodevelopmental traits, mental health, empathetic attitudes

\section{Introduction}

In recent years, the critical effects of depression on the empathy, professional behavior, and performance of medical students have been recognized globally. ${ }^{1}$ A systematic review reported that the prevalence of depression among medical students was $27.2 \%$, similar to that among residents, and significantly higher than that of the general population matched for age. ${ }^{2}$ Despite the limited research on the prevalence of mental disorders other than depression in medical students, about $30 \%$ of medical students who reported having disabilities in the United States are
Correspondence: Takafumi Watanabe Department of Psychiatry, Nagoya City University Graduate School of Medical Sciences, I, Kawasumi, Mizuho-Cho, Mizuho-Ku, Nagoya City, Aichi, 467-860I, Japan

Tel +8I 528538271

$\mathrm{Fax}+81528520837$

Email tw28@med.nagoya-cu.ac.jp 
reported to have attention-deficit/hyperactivity disorder (ADHD). ${ }^{3}$ According to an early intervention report by a mental healthcare team, $20 \%$ of the medical students they cared for were diagnosed with ADHD, followed by depression, anxiety, and adjustment disorders in frequency. ${ }^{4} \mathrm{~A}$ small Paraguayan study reported that 3\% of their medical students had ADHD or autism spectrum disorder (ASD). ${ }^{5}$ Individuals with ADHD and ASD are assumed to lie on a continuum with typically developing individuals. Moreover, it has been observed that some atypically developing individuals are close to the diagnostic threshold for ADHD and/or ASD, experience few behavioral problems, display good cognitive function, and perform well, while others demonstrate various psychiatric disorders. ${ }^{6}$ In Japanese hospital pharmacists, ADHD and ASD traits have been reported to be associated with psychological distress, burnout, and compassion fatigue. $^{7}$

A major mission of medical education is to educate physicians to express compassion and empathy toward their patients. Empathy in physicians within patient care settings is defined as the cognitive ability to understand a patient's inner experiences and perspectives and to communicate that understanding. ${ }^{8}$ Empathy has reported correlations with medical students' clinical competency, and it is a core competency required in their clinical clerkships. ${ }^{9}$ However, medical students are regularly exposed to challenging academic demands and emotionally exhausting work environments. As a result, some evidence indicates a decline in empathy during clinical clerkships, thereby impairing their ability to communicate with patients and their overall competence. ${ }^{10,11}$

However, existing studies have not examined the associations of ADHD and ASD traits with depression and empathy among medical students. Thus, this study aimed to investigate whether ADHD and ASD traits are associated with depression and empathy among Japanese medical students.

\section{Patients and Methods}

\section{Study Design and Participants}

In Japan, medical education requires a 6-year undergraduate program, with the last 2 years devoted to clinical clerkships. A cross-sectional survey was conducted among fifth-year students at a medical school. The survey was implemented twice in November 2019 and 2020, for 10 months after the students started their clinical clerkship. A total of 202 students were invited to participate in an online survey using Google Forms. They were provided with a gift certificate of 500 yen as an incentive to complete the survey. This study was conducted according to the Strengthening the Reporting of Observational Studies in Epidemiology (STROBE) statement guidelines. All respondents provided written consent before completing the survey.

\section{Measures}

\section{Depression}

In this study, the Hospital Anxiety and Depression Scale (HADS), developed for clinical populations, was employed to measure psychological distress. ${ }^{12}$ This scale has been widely used in previous studies among students in general and medical students. ${ }^{2,13,14}$ The HADS comprises two subscales - on anxiety and depression - including seven items each with a 4-point Likert scale. Each subscale score ranged from $0-21$, and the total score ranged from $0-42$. A higher total score indicated greater psychological distress. The psychometric properties of the Japanese version of the questionnaire have been validated. The cutoff points of the HADS total score for screening major depressive disorder and adjustment disorder were $19 / 20$ (sensitivity $82.4 \%$, specificity $96.3 \%$ ) and $10 / 11$ (sensitivity $91.5 \%$, specificity $65.4 \%$ ), respectively, ${ }^{15}$ In the present study, medical students with a total score of $\geq 20$ on the HADS were categorized as depressed.

\section{Empathy}

The Jefferson Scale of Empathy-Student version (JSE) was used to measure empathy between medical students and patients. ${ }^{16}$ The JSE is a 20-item Likert-type selfadministered questionnaire that assesses empathetic attitudes of healthcare professionals within patient care settings. The total scores on this scale ranged from 20-140. The scale measures three aspects of empathy-perspective-taking, compassionate care, and the ability to stand in the patient's shoes - where higher scores indicate greater empathy. The Japanese version of this scale has been psychometrically validated. $^{17}$

\section{ADHD and ASD Traits}

The World Health Organization has developed the Adult ADHD Self-Report Scale (ASRS) as a screening tool for adult ADHD and translated it into various languages, including Japanese. In accordance with the Diagnostic and Statistical Manual of Mental Disorders-Fourth Edition's ADHD criteria, ASRS has 6 and 12 items in 
Part A (ASRS-Screener) and B, respectively. The ASRSScreener comprises six items that predict ADHD symptoms most accurately and meeting four or more of the six items is considered to be highly consistent with an adult ADHD diagnosis. ${ }^{18}$

The Autism Spectrum Quotient (AQ) is a 50-item selfadministered scale, developed by Baron-Cohen et al, ${ }^{19}$ which is used worldwide to measure the degree of ASD traits present in adults with normal-range intelligence scores. Two Japanese versions of this scale have been psychometrically validated. ${ }^{20,21}$ A shortened version of the Japanese version of the AQ (AQ-J) - AQ-J-21 - was developed by Kurita et al, ${ }^{20}$ which reported a higher sensitivity and specificity than the 50-item AQ-J. The cutoff point of the AQ-J-21 total score was 12 points for distinguishing between patients with ASD and a control group selected from the general population.

\section{Demographic and Occupational Characteristics}

Demographic information regarding participants' gender, age, marital and parental status, cohabitants, extracurricular activities, prior enrollment in other academic departments, part-time jobs, and alcohol consumption (more than three drinks per week) was obtained. Marital and parental status were excluded from the analysis because only one student was married and parenting children with the same person.

\section{Statistical Analysis}

Descriptive statistics were computed for the study sample, including absolute and relative frequencies (\%), mean, and standard deviation (SD). Furthermore, students with and without depression were statistically compared using a bivariate analysis. Categorical variables were assessed using the $\chi^{2}$ test, while continuous variables were assessed using the $t$-test (normal distribution) and Mann-Whitney $U$-test (non-normal distribution). Multivariate linear regression analyses were used to examine the association of ADHD and ASD traits with depression and empathy while controlling for potential covariates. ${ }^{22}$ The following variables were incorporated into the models: gender (categorical), age (continuous), and ADHD and ASD trait scores evaluated by the ASRS Screener and AQ-J-21 (continuous). All statistical analyses were performed using EZR (Saitama Medical Center, Jichi Medical University), which is a graphical user interface for $\mathrm{R}$ (The R Foundation for Statistical Computing, Vienna,
Austria), with statistical functions frequently used in biostatistics. Statistical significance was set at $\mathrm{p}<0.05$.

\section{Results}

Among the 202 fifth-year medical students, 151 completed the online survey. Of these, $52(34.4 \%)$ were women, and the mean age was 23.7 years (SD: 1.67) (Table 1). Participants' mean total score on the HADS was 15.21 (SD: 6.53). Depression was screened among 27.2\% (n= $41,95 \%$ confidence interval [CI]: 20.2-35.0) of the medical student participants.

Accordingly, participant characteristics were compared between the two participant groups: depressed and nondepressed medical students (Table 2). Compared to nondepressed medical students, depressed medical students were significantly less likely to have part-time jobs and were more likely to be enrolled in other faculties. Moreover, depressed medical students' mean scores were significantly higher than

Table I Demographic Characteristics and Descriptive Statistical Data of Medical Students

\begin{tabular}{|c|c|}
\hline & $n=|5|$ \\
\hline & Number $(\%, 95 \% \mathrm{Cl})$ \\
\hline Gender (female) & $52(34.4,26.9-42.6)$ \\
\hline $\begin{array}{l}\text { Alcohol consumption more than } 3 \\
\text { times/week }\end{array}$ & $22(|4.6,9.4-2| .2)$ \\
\hline Participation in extracurricular activities & $102(67.5,59.5-74.9)$ \\
\hline Living alone & $54(35.8,28.1-44.0)$ \\
\hline History of enrollment in other faculties & $7(4.6,1.9-9.3)$ \\
\hline A part-time employment & $104(68.9,60.8-76.2)$ \\
\hline With depression * & $4 \mid(27.2,20.2-35.0)$ \\
\hline With ADHD traits $* *$ & $31(20.5,14.4-27.9)$ \\
\hline \multirow[t]{2}{*}{ With ASD traits $* * *$} & $42(27.8,20.8-35.7)$ \\
\hline & Mean (SD) \\
\hline Age (years) & $23.70(1.67)$ \\
\hline ASRS Screener & $2.30(1.62)$ \\
\hline AQ-J-2I & $9.30(3.83)$ \\
\hline HADS total & $|5.2|(6.53)$ \\
\hline JSE & $101.06(\mid 4.80)$ \\
\hline
\end{tabular}

Notes: *HADS score of 20 or higher was defined as having depression, **ASRS screener score of 4 or higher was defined as having ADHD traits, ***AQ-J-2I score of 12 or higher was defined as having ASD traits.

Abbreviations: ADHD, attention-deficit/hyperactivity disorder; AQ-J, AutismSpectrum Quotient, Japanese version; ASD, autism spectrum disorder; ASRS, Adult ADHD Self Report Scale; HADS, Hospital Anxiety and Depression Scale; JSE, Jefferson Scale of Empathy-Student version; Cl, confidence interval; SD, standard deviation. 
Table 2 Demographic Characteristics and Descriptive Statistical Data of Medical Students Grouped by the Presence of Depression*

\begin{tabular}{|c|c|c|c|}
\hline & $\begin{array}{l}\text { Student without Depression } \\
\qquad(\mathrm{N}=|\mathrm{I}| 0)\end{array}$ & $\begin{array}{l}\text { Student with Depression } \\
\qquad(\mathrm{N}=4 \mathrm{I})\end{array}$ & Significance Tests** \\
\hline & Mean (SD) & Mean (SD) & \\
\hline Gender (female) & $42(38.2)$ & $10(24.4)$ & $F(I)=1.94, p$-value $=0.16$ \\
\hline $\begin{array}{l}\text { Alcohol consumption more than } 3 \\
\text { times/week }\end{array}$ & $14(12.7)$ & $8(19.5)$ & $F(I)=0.63, p$-value $=0.43$ \\
\hline $\begin{array}{l}\text { Participation in extracurricular } \\
\text { activities }\end{array}$ & $78(70.9)$ & $24(58.5)$ & $F(I)=1.56, p$-value $=0.21$ \\
\hline Living alone & $71(64.5)$ & $26(63.4)$ & $F(I)=0.00, p$-value $=I$ \\
\hline Presence of a part-time job & $84(76.4)$ & $20(48.8)$ & $F(I)=9.35, p$-value $=0.002 * * *$ \\
\hline \multirow{2}{*}{$\begin{array}{l}\text { History of enrollment in other } \\
\text { faculties }\end{array}$} & $0(0.0)$ & $7(17.1)$ & $F(I)=16.021, p$-value $=0.000^{* * *}$ \\
\hline & Number (\%) & Number (\%) & \\
\hline Age & $23.41(1.03)$ & $24.46(2.58)$ & $U=1825.5, p$-value $=0.059$ \\
\hline ASRS Screener & $1.98(1.54)$ & $3.15(1.56)$ & $U=1319, p$-value $=0.000^{* * *}$ \\
\hline AQ-J-2I & $8.89(3.92)$ & $10.39(3.39)$ & $\mathrm{t}=-2.1639, \mathrm{p}$-value $=0.032 * * *$ \\
\hline JSE & $104.07(14.04)$ & $92.98(13.87)$ & $U=3225.5, p$-value $=0.000 * * *$ \\
\hline
\end{tabular}

Notes: *Hospital Anxiety and Depression Scale score of 20 or higher was defined as depression; **Chi-squared tests for categorical data. Mann-Whitney U-test or $t$-test for numerical data; $* * * \mathrm{p}<0.05$.

Abbreviations: ASRS, Adult ADHD Self Report Scale; AQ-J, Autism-Spectrum Quotient, Japanese version; HADS, Hospital Anxiety and Depression Scale; JSE, Jefferson Scale of Empathy-Student version; SD, standard deviation.

that of non-depressed medical students for the ASRS Screener and AQ-J-21 and significantly lower for the JSE.

\section{Associations of ADHD and ASD Traits with Depression and Empathy in Japanese Medical Students}

Based on the ASRS and AQ-J-21 cutoff values, the prevalence rates of ADHD and ASD traits were $20.5 \%(\mathrm{n}=31,95 \% \mathrm{CI}$ : 14.4-27.9) and $27.8 \%(\mathrm{n}=42,95 \%$ CI: $20.8-35.7)$, respectively. Furthermore, we controlled for the covariates of gender and age and performed multiple linear regression analysis to examine the association of ADHD and ASD traits with depression, as evaluated by the HADS total score (Table 3). We obtained a model that explained $24.5 \%$ of the variance. This model reported that age ( $\beta=0.85,95 \%$ CI: $0.29-1.42$ ), ASRS Screener score ( $\beta=1.10,95 \%$ CI: 0.48-1.72), and AQ-J-21 score $(\beta=0.40,95 \%$ CI: $0.14-0.66)$ significantly predicted the HADS total score. Similarly, another multiple regression analysis was performed, controlling for gender and age as covariates, to examine the association of ADHD and ASD traits with empathy, as assessed using the JSE score (Table 4). We obtained a model that explained $15.1 \%$ of the variance. In
Table 3 Multiple Linear Regression Analysis Between Depression and ADHD/ASD Traits, Adjusted by Gender and Age

\begin{tabular}{|l|l|c|c|}
\hline & & $\boldsymbol{\beta}(\mathbf{9 5} \% \mathbf{C l}$ ) & Multiple $\mathbf{R}^{2}$ \\
\hline HADS & Constant & $-11.78(-25.29,1.73)$ & 0.245 \\
& Gender & $0.69(-1.29,2.66)$ & \\
& Age & $\mathbf{0 . 8 5}(\mathbf{0 . 2 9}, 1.42)$ & \\
& ASRS Screener & $\mathbf{1 . 1 0 ( 0 . 4 8 , 1 . 7 2 )}$ & \\
& AQ-J-2I & $\mathbf{0 . 4 0 ( 0 . 1 4 , 0 . 6 6 )}$ & \\
\hline
\end{tabular}

Note: Bold text indicates statistical significance at $\mathrm{p}<0.05$.

Abbreviations: ADHD, attention-deficit/hyperactivity disorder; AQ-J, AutismSpectrum Quotient, Japanese version; ASD, autism spectrum disorder; ASRS, Adult ADHD Self Report Scale; $\mathrm{Cl}$, confidence interval; HADS, Hospital Anxiety and Depression Scale.

this model, the AQ-J-21 score $(\beta=-1.31,95 \%$ CI: -2.00 to $-0.77)$ significantly predicted the JSE score.

\section{Discussion}

In this study, $27.2 \%$ of fifth-year medical student participants $(n=151)$ reported total HADS scores above the depression cutoff criteria, at 10 months after the start of their clinical clerkship. Medical students with severe depression worked part-time jobs less often, apart from their studies, and were more often enrolled in other 
Table 4 Multiple Linear Regression Analysis Between Empathy and ADHD/ASD Traits, Adjusted by Gender and Age

\begin{tabular}{|l|l|c|c|}
\hline & & $\boldsymbol{\beta}(\mathbf{9 5 \%}$ CI*) & Multiple $\mathbf{R}^{\mathbf{2}}$ \\
\hline \multirow{3}{*}{ JSE } & Constant & $126.3 \mathrm{I}(94.25,158.36)$ & $0.15 \mathrm{I}$ \\
& Gender & $-0.99(-5.67,3.69)$ & \\
& Age & $-0.40(-1.74,0.94)$ & \\
& ASRS Screener & $-0.98235(-2.44,0.47)$ & \\
& AQ-J-2 I & $-1.38(-\mathbf{2 . 0 0},-\mathbf{0 . 7 7})$ & \\
\hline
\end{tabular}

Note: Bold text indicates statistical significance at $p<0.05$.

Abbreviations: ADHD, attention-deficit/hyperactivity disorder; AQ-J, AutismSpectrum Quotient, Japanese version; ASD, Autism Spectrum Disorder; ASRS, Adult ADHD Self Report Scale; $\mathrm{Cl}$, confidence interval; JSE, Jefferson Scale of Empathy-Student version.

faculties previously. Multiple regression analysis revealed that higher age and the degree of ADHD and ASD traits had a significant positive association with the severity of depression. However, empathy for the patients, as evaluated by the JSE scores, reported a significant negative association only with the degree of ASD traits.

A nationwide Norwegian population-based study suggested that adults with ADHD and/or ASD reported a 33.7 times higher prevalence of major depressive disorder than adults without either. ${ }^{23}$ Despite the existing research on the association of ADHD and ASD traits with psychological distress among hospital pharmacists, there have been no previous reports examining the association of the degree of ADHD and ASD traits with depression in medical students. ${ }^{7}$ The current study results suggest that the degree of ADHD and ASD traits may be a risk factor for depression among medical students. In addition, this study found associations between older age and depression. Existing reports have observed an association between depression and grade level in medical students, but not with student ages in the same academic year. ${ }^{24-26}$ In Japan, several medical students enter medical school immediately after high school graduation, thus same year medical students' age is almost uniform. However, many older medical students either transfer from other faculties or have to repeat their school years. As a result, older medical students might be more likely to be isolated and depressed. Therefore, medical students with these characteristics need to be treated cautiously for depression. Furthermore, a higher degree of ASD traits was associated with lower levels of empathy in the present study. This result was consistent with a previous study among Japanese pharmacy students. ${ }^{27}$ Among medical students with strong ASD traits, some interventions are particularly needed for depression prevention and improved empathy.
Despite the effectiveness of communication skills and mindfulness training for improving empathy in medical students, ${ }^{28-30}$ developing interventions for medical students with ASD traits continues to remain a challenge. Among the AQ subscales (social skills, attention switching, attention to detail, communication, and imagination), social skills significantly predicted decreased empathy and helping motivation in adults with ASD. ${ }^{31}$ Moreover, behavioral therapy interventions, such as theory of mind training, have demonstrated increased empathy in children with autism. $^{32,33}$ Similarly, mindfulness-based therapy and acceptance and commitment therapy have also demonstrated effectiveness in alleviating depressive symptoms in adults with ASD. ${ }^{34,35}$

This study has several limitations. First, this was a cross-sectional study, and a causal relationship could not be determined between the surveyed factors-ADHD and ASD traits, depression, and empathy. However, ADHD and ASD represent developmental traits and therefore logically antedate depression; therefore, these traits may then still not be causal factors but can at least be predictors of depression. Second, this study used selfadministered questionnaires, which may have caused information bias. Despite the validation of these questionnaires, a definitive diagnosis could not be established. Third, a potential selection bias may have arisen because the questionnaires were not answered by all participants. However, this bias may be very small, as almost $75 \%$ of the current study participants answered the questionnaires. Lastly, this was a single-center study, and the hospital, where this study was conducted, is similar to other physician training institutions in Japan. However, it is necessary to conduct similar studies at other institutions in the future to ensure the accurate generalization of these findings.

\section{Conclusion}

This is the first study to explore the associations of neurodevelopmental traits with depression or empathy among medical students. About $30 \%$ of the medical students in Japan scored above the screening threshold for major depressive disorder during their clinical clerkship. Older age and a higher degree of ADHD and ASD traits were positively associated with the severity of depression. Furthermore, ASD traits are also associated with lower empathy for their patients. Therefore, intervention strategies should be urgently developed to accommodate the worsening depression and lower empathy among medical students with these traits. 


\section{Abbreviations}

ADHD, attention-deficit/hyperactivity disorder; AQ-J, Autism-Spectrum Quotient, Japanese version; ASD, autism spectrum disorder; ASRS, Adult ADHD Self Report Scale; CI, confidence interval; HADS, Hospital Anxiety and Depression Scale; IRB, Institutional Review Board; JSE, Jefferson Scale of Empathy-Student version; SD, standard deviation.

\section{Ethical Considerations}

All participants were informed of the purpose of this study. This research was conducted in accordance with the principles of the Declaration of Helsinki. The Institutional Review Boards (IRB) at the Nagoya City University of Medical Science and the Nagoya City University Graduate School of Medicine approved the study and all its scientific and ethical aspects, respectively. The authors complied with all IRB recommendations to maintain the confidentiality and anonymity of the participants.

\section{Acknowledgments}

The authors appreciate the participation of the medical students at the Nagoya City University Medical School. We also appreciate Ms. Kaori Kobori, Secretary, Department of Psychiatry, Nagoya City University Graduate School of Medicine, for her cooperation in this study. We are grateful to Editage (www.editage.com) for the English editing.

\section{Author Contributions}

TW, MK, MS, and ST were involved in the conception and design of this study. TW, MK, MS, and ST acquired the data. TW, MK, TAF, and AT analyzed and interpreted the data. TW drafted the manuscript. TAF and AK contributed substantially to the revision of the manuscript. All authors read and approved the final version of the manuscript. All authors contributed to data analysis, drafting or revising the article, have agreed on the journal to which the article will be submitted, gave final approval of the version to be published, and agree to be accountable for all aspects of the work.

\section{Funding}

This study was partially funded by the Nagoya City University Graduate School of Medical Sciences under grant number 2113020. The funders had no involvement in the design of the study, collection, analysis, and interpretation of data, or in writing the manuscript.

\section{Disclosure}

MK reports personal fees from Shionogi \& Co., Ltd. and Yoshitomiyakuhin Corporation, outside the submitted work. TAF reports grants and personal fees from Mitsubishi-Tanabe, personal fees from MSD, personal fees from SONY, grants and personal fees from Shionogi, outside the submitted work. In addition, TAF has a patent 2020-548587 concerning smartphone CBT apps pending, and intellectual properties for Kokoro-app licensed to Mitsubishi-Tanabe. AK has received lectures fees from AstraZeneca, Chugai, Daiichi-Sankyo, Dainippon-Sumitomo, Eisai, Hisamitsu, Janssen, Kyowa, Kyowa Kirin, Lilly, MSD, Meiji-Seika Pharma, Mochida, Mundipharma, Nipro, Nihon-Zoki, Otsuka, Pfizer, Takeda, Tsumura, and Viatris. He has received royalties from Igakushoin and research funds from Daiichi-Sankyo, Eisai, Fujifilm RI Pharma, MSD, Otsuka, and Shionogi. AK is the inventor of the pending patents (2019-017498 \& 2020-135195). The authors report no other conflicts of interest in this work.

\section{References}

1. Gold JA, Hu X, Huang G, et al. Medical student depression and its correlates across three international medical schools. World J Psychiatry. 2019;9(4):65-77. doi:10.5498/wjp.v9.i4.65

2. Rotenstein LS, Ramos MA, Torre M, et al. Prevalence of depression, depressive symptoms, and suicidal ideation among medical students: a systematic review and meta-analysis. JAMA. 2016;316 (21):2214-2236. doi:10.1001/jama.2016.17324

3. Meeks LM, Case B, Herzer K, Plegue M, Swenor BK. Change in prevalence of disabilities and accommodation practices among US medical schools, 2016 vs 2019. JAMA. 2019;322(20):2022-2024. doi:10.1001/jama.2019.15372

4. Karp JF, Levine AS. Mental health services for medical students time to act. $N$ Engl J Med. 2018;379(13):1196-1198. doi:10.1056/ NEJMp1803970

5. Torales J, Kadhum M, Zárate G, et al. Wellbeing and mental health among medical students in Paraguay. Int Rev Psychiatry. 2019;31(78):598-602. doi:10.1080/09540261.2019.1667172

6. Pehlivanidis A, Papanikolaou K, Mantas V, et al. Lifetime co-occurring psychiatric disorders in newly diagnosed adults with attention deficit hyperactivity disorder (ADHD) or/and autism spectrum disorder (ASD). BMC Psychiatry. 2020;20(1):423. doi:10.1186/ s12888-020-02828-1

7. Higuchi $Y$, Inagaki M, Koyama T, et al. A cross-sectional study of psychological distress, burnout, and the associated risk factors in hospital pharmacists in Japan. BMC Public Health. 2016;16:534. doi:10.1186/s12889-016-3208-5

8. Hojat M, Gonnella JS, Nasca TJ, Mangione S, Vergare M, Magee M. Physician empathy: definition, components, measurement, and relationship to gender and specialty. Am J Psychiatry. 2002;159 (9):1563-1569. doi:10.1176/appi.ajp.159.9.1563

9. Hojat M, Gonnella JS, Mangione S, et al. Empathy in medical students as related to academic performance, clinical competence and gender. Med Educ. 2002;36(6):522-527. doi:10.1046/j.13652923.2002.01234.x

10. Hojat M, Vergare MJ, Maxwell K, et al. The devil is in the third year: a longitudinal study of erosion of empathy in medical school. Acad Med. 2009;84(9):1182-1191. doi:10.1097/ACM.0b013e3181b17e55 
11. Thomas MR, Dyrbye LN, Huntington JL, et al. How do distress and well-being relate to medical student empathy? A multicenter study. J Gen Intern Med. 2007;22(2):177-183. doi:10.1007/s11606-0060039-6

12. Zigmond AS, Snaith RP. The hospital anxiety and depression scale. Acta Psychiatr Scand. 1983;67(6):361-370. doi:10.1111/j.16000447.1983.tb09716.x

13. Matsudaira T, Igarashi H, Kikuchi H, et al. Factor structure of the hospital anxiety and depression scale in Japanese psychiatric outpatient and student populations. Health Qual Life Outcomes. 2009;7 (1):42. doi:10.1186/1477-7525-7-42

14. Kotter T, Tautphaus Y, Scherer M, Voltmer E. Health-promoting factors in medical students and students of science, technology, engineering, and mathematics: design and baseline results of a comparative longitudinal study. BMC Med Educ. 2014;14:134. doi:10.1186/1472-6920-14-134

15. Kugaya A, Akechi T, Okuyama T, Okamura H, Uchitomi Y. Screening for psychological distress in Japanese cancer patients. Jpn J Clin Oncol. 1998;28(5):333-338. doi:10.1093/jjco/28.5.333

16. Hojat M, Mangione S, Nasca TJ, et al. The Jefferson scale of physician empathy: development and preliminary psychometric data. Educ Psychol Meas. 2001;61(2):349-365. doi:10.1177/ 00131640121971158

17. Kataoka HU, Koide N, Ochi K, Hojat M, Gonnella JS. Measurement of empathy among Japanese medical students: psychometrics and score differences by gender and level of medical education. Acad Med. 2009;84(9):1192-1197. doi:10.1097/ACM.0b013e3181b180d4

18. Kessler RC, Adler L, Ames M, et al. The World Health Organization Adult ADHD Self-Report Scale (ASRS): a short screening scale for use in the general population. Psychol Med. 2005;35(2):245-256. doi: $10.1017 / \mathrm{s} 0033291704002892$

19. Baron-Cohen S, Wheelwright S, Skinner R, Martin J, Clubley E. The Autism-Spectrum Quotient (AQ): evidence from Asperger syndrome/ high-functioning autism, males and females, scientists and mathematicians. J Autism Dev Disord. 2001;31(1):5-17. doi:10.102 3/a:1005653411471

20. Kurita H, Koyama T, Osada H. Autism-Spectrum Quotient-Japanese version and its short forms for screening normally intelligent persons with pervasive developmental disorders. Psychiatry Clin Neurosci. 2005;59(4):490-496. doi:10.1111/j.1440-1819.2005.01403.x

21. Wakabayashi A, Baron-Cohen S, Wheelwright S, Tojo Y. The Autism-Spectrum Quotient (AQ) in Japan: a cross-cultural comparison. J Autism Dev Disord. 2006;36(2):263-270. doi:10.100 7/s10803-005-0061-2

22. Westreich D, Greenland S. The table 2 fallacy: presenting and interpreting confounder and modifier coefficients. Am J Epidemiol. 2013;177(4):292-298. doi:10.1093/aje/kws412

23. Solberg BS, Zayats T, Posserud MB, et al. Patterns of psychiatric comorbidity and genetic correlations provide new insights into differences between attention-deficit/hyperactivity disorder and autism spectrum disorder. Biol Psychiatry. 2019;86(8):587-598. doi:10.10 16/j.biopsych.2019.04.021
24. Tam W, Lo K, Pacheco J. Prevalence of depressive symptoms among medical students: overview of systematic reviews. Med Educ. 2019;53(4):345-354. doi:10.1111/medu.13770

25. Mahroon ZA, Borgan SM, Kamel C, Maddison W, Royston M, Donnellan C. Factors associated with depression and anxiety symptoms among medical students in Bahrain. Acad Psychiatry. 2018;42 (1):31-40. doi:10.1007/s40596-017-0733-1

26. Ngasa SN, Sama CB, Dzekem BS, et al. Prevalence and factors associated with depression among medical students in Cameroon: a cross-sectional study. BMC Psychiatry. 2017;17(1):216. doi:10.1186/ s12888-017-1382-3

27. Higuchi Y, Inagaki M, Koyama T, et al. Emotional intelligence and its effect on pharmacists and pharmacy students with autistic-like traits. Am J Pharm Educ. 2017;81(4):74. doi:10.5688/ajpe81474

28. Winter R, Issa E, Roberts N, Norman RI, Howick J. Assessing the effect of empathy-enhancing interventions in health education and training: a systematic review of randomised controlled trials. BMJ. 2020;10(9):e036471. doi:10.1136/bmjopen-2019-036471

29. Barbosa P, Raymond G, Zlotnick C, Wilk J, Toomey III R, Mitchell III J. Mindfulness-based stress reduction training is associated with greater empathy and reduced anxiety for graduate healthcare students. Educ Health. 2013;26(1):9-14. doi:10.4103/13576283.112794

30. Krasner MS, Epstein RM, Beckman H, et al. Association of an educational program in mindful communication with burnout, empathy, and attitudes among primary care physicians. JAMA. 2009;302 (12):1284-1293. doi:10.1001/jama.2009.1384

31. Komeda H, Kosaka H, Fujioka T, Jung M, Okazawa H. Do individuals with autism spectrum disorders help other people with autism spectrum disorders? An investigation of empathy and helping motivation in adults with autism spectrum disorder. Front Psychiatry. 2019;10:376. doi:10.3389/fpsyt.2019.00376

32. Holopainen A, de Veld DMJ, Hoddenbach E, Begeer S. Does theory of mind training enhance empathy in autism? J Autism Dev Disord. 2019;49(10):3965-3972. doi:10.1007/s10803-018-3671-1

33. Schrandt JA, Townsend DB, Poulson CL. Teaching empathy skills to children with autism. J Appl Behav Anal. 2009;42(1):17-32. doi:10.1901/jaba.2009.42-17

34. Spek AA, van Ham NC, Nyklíček I. Mindfulness-based therapy in adults with an autism spectrum disorder: a randomized controlled trial. Res Dev Disabil. 2013;34(1):246-253. doi:10.1016/j. ridd.2012.08.009

35. Byrne G, O’Mahony T. Acceptance and commitment therapy (ACT) for adults with intellectual disabilities and/or autism spectrum conditions (ASC): a systematic review. $J$ Contextual Behav Sci. 2020;18:247-255. doi:10.1016/j.jcbs.2020.10.001
Advances in Medical Education and Practice

\section{Publish your work in this journal}

Advances in Medical Education and Practice is an international, peerreviewed, open access journal that aims to present and publish research on Medical Education covering medical, dental, nursing and allied health care professional education. The journal covers undergraduate education, postgraduate training and continuing medical education including emerging trends and innovative models linking education, research, and health care services. The manuscript management system is completely online and includes a very quick and fair peer-review system. Visit http://www.dovepress.com/testimonials.php to read real quotes from published authors. 